\title{
Quando os Alunos Perguntam
}

\section{Carlos CorrêA*}

Todos os professores sabem bem que os alunos contribuem para a sua formação, em especial pela curiosidade que demonstram e exprimem nas perguntas que nos fazem. Aconteceu algumas vezes que a nossa resposta foi simplesmente "engraçado, nunca tinha pensado nisso, mas vou pensar para Ihe dar a resposta".

Neste artigo analisam-se algumas perguntas e respostas relativas a alguns casos que nos parecem ter interesse para os professores de Química.

\section{As PERGUNTAS}

\section{Poder Redutor dos Metals ALCALINOS}

Olhando a posição dos elementos na Tabela Periódica e atendendo às correspondentes energias de ionização, verifica-se que a dificuldade para remover um electrão

$$
\mathrm{M}(\mathrm{g}) \rightarrow \mathrm{M}^{+}(\mathrm{g})+\mathrm{e}^{-}
$$

diminui ao longo do grupo, ou seja, o poder redutor dos metais alcalinos cresce com $Z$ ao longo do grupo. Apesar disto, os potenciais de eléctrodo correspondentes aos pares conjugados oxido-redutores não seguem a mesma ordem [1]:

$E^{\circ}\left(\mathrm{Li}^{+} / \mathrm{Li}\right)=-3,04 \mathrm{~V}<E^{\circ}\left(\mathrm{K}^{+} / \mathrm{K}\right)=$ $-2,924 \mathrm{~V}<E^{\circ}\left(\mathrm{Na}^{+} / \mathrm{Na}\right)=-2,713 \mathrm{~V}$

ou seja, o lítio é um redutor mais forte que o sódio e o potássio!

Por que será?

\section{Catiões cobre(I) e cobre(II)}

O catião cobre(II) é bem conhecido dos alunos, o que não sucede com o catião $\mathrm{Cu}(\mathrm{I})$.

As configurações electrónicas:

$$
\begin{aligned}
& \mathrm{Cu}-1 s^{2}-2 s^{2} 2 p^{6}-3 s^{2} 3 p^{6} 3 d^{10}-4 s^{1} \\
& C u^{+}-1 s^{2}-2 s^{2} 2 p^{6}-3 s^{2} 3 p^{6} 3 d^{10} \\
& \mathrm{Cu}^{2+}-1 s^{2}-2 s^{2} 2 p^{6}-3 s^{2} 3 p^{6} 3 d^{9}
\end{aligned}
$$

\footnotetext{
Professor Catedrático Jubilado do Departamento de Química da Faculdade de Ciências da Universidade do Porto
}

indicam que o catião $\mathrm{Cu}(\mathrm{I})$ deveria ser mais estável que o catião $\mathrm{Cu}(\mathrm{II})$, o que é confirmado pelas sucessivas energias de ionização [1]:

$$
\begin{aligned}
& I_{1}=745,4 \mathrm{~kJ} / \mathrm{mol} \\
& I_{2}=1958 \mathrm{~kJ} / \mathrm{mol}
\end{aligned}
$$

Apesar disto, o catião $\mathrm{Cu}(\mathrm{I})$ é instável e dismuta rapidamente em solução aquosa, dando o catião $\mathrm{Cu}(\mathrm{II}) \mathrm{e}$ $\mathrm{Cu}(\mathrm{s})$.

$$
2 \mathrm{Cu}^{+}(\mathrm{aq}) \rightarrow \mathrm{Cu}^{2+}(\mathrm{aq})+\mathrm{Cu}(\mathrm{s})
$$

\section{Qual a razão?}

\section{A estabilidade da estrutura DE GASES} NOBRES

É vulgar referir-se a tendência para os átomos adquirirem uma configuração electrónica de gás nobre, captando ou perdendo os electrões necessários. Assim, diz-se que os metais alcalinos, com um só electrão na última camada, tendem a perder esse electrão $s$ para adquirir a configuração electrónica de gás nobre, o que explica a vulgaridade dos catiões monopositivos dos metais alcalinos.

No entanto, se repararmos nas correspondentes energias de ionização e afinidades electrónicas dos metais alcalinos e do hidrogénio [1] não se vislumbra qualquer tendência para perder electrões e adquirir a estrutura electrónica de gás nobre. Pelo contrário, todos eles têm maior tendência para captar electrões, dando aniões!

A dificuldade para remover o electrão $n^{1}$ e originar um ião monopositivo com configuração electrónica de gás nobre é sempre da ordem de meio milhar de kilojoules por mole, enquanto a transformação num ião mononegativo é energeticamente mais favorável!

\begin{tabular}{|l|l|r|r|}
\hline \multicolumn{1}{|c|}{ Elemento } & Configuração electrónica & $\begin{array}{c}1^{\mathrm{a}} \text { energia de } \\
\text { ionização } \\
(\mathrm{kJ} / \mathrm{mol})\end{array}$ & $\begin{array}{c}\text { Afinidade } \\
\text { electrónica } \\
(\mathrm{kJ} / \mathrm{mol})\end{array}$ \\
\hline $\mathrm{Li}$ & $1 \mathrm{~s}^{2}-2 \mathrm{~s}^{1}$ & 513,3 & 59,6 \\
\hline $\mathrm{Na}$ & $1 \mathrm{~s}^{2}-2 \mathrm{~s}^{2} 2 \mathrm{p}^{6}-3 \mathrm{~s}^{1}$ & 495,8 & 52,9 \\
\hline $\mathrm{K}$ & $1 \mathrm{~s}^{2}-2 \mathrm{~s}^{2} 2 \mathrm{p}^{6}-3 \mathrm{~s}^{2} 3 \mathrm{p}^{6}-4 \mathrm{~s}^{1}$ & 418,8 & 48,2 \\
\hline $\mathrm{H}$ & $1 \mathrm{~s}^{1}$ & 1312 & 72,8 \\
\hline
\end{tabular}



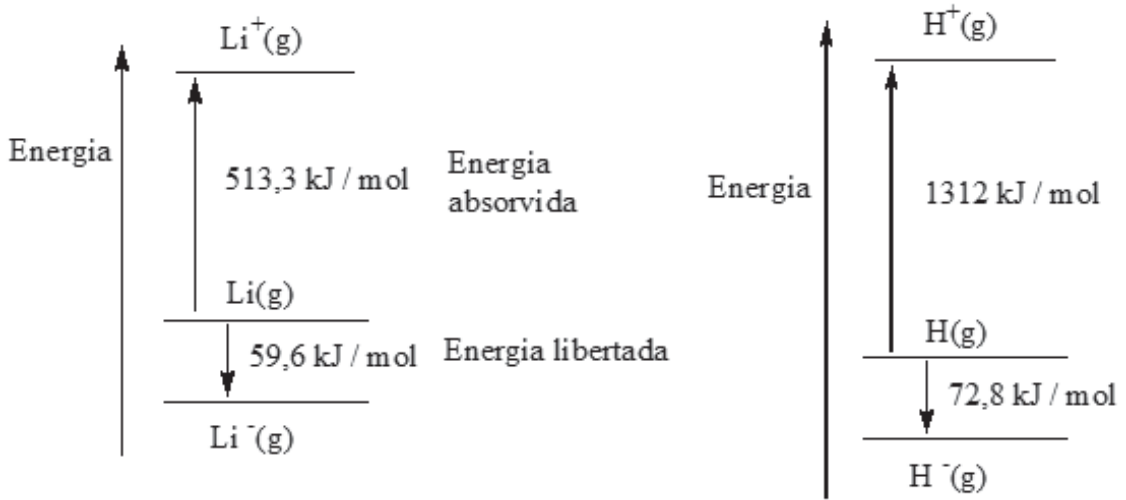

Cabe perguntar se os aniões $\mathrm{Li}^{-}, \mathrm{Na}^{-}$, $\mathrm{K}^{-}$e $\mathrm{H}^{-}$não se podem formar e qual a razão de os catiões serem muito mais familiares.

\section{As RESPOSTAS}

Todas as respostas se baseiam na diferença entre o que se passa em fase gasosa e em solução ou no estado sólido. As energias de ionização dos átomos (relacionadas com a estabilidade das configurações electrónicas) referem-se aos átomos no estado gasoso e, quando em solução ou no estado sólido, as interacções com o solvente ou com outros átomos vão alterar o comportamento das substâncias.

\section{Poder Redutor dos metais alcalinos} [2a]

Uma reacção de oxidação-redução do tipo

$$
\mathrm{M}(\mathrm{s})+\mathrm{N}^{+}(\mathrm{aq}) \rightarrow \mathrm{M}^{+}(\mathrm{aq})+\mathrm{N}(\mathrm{s})
$$

será tanto mais extensa quanto maior for a diferença entre os potenciais normais de eléctrodo, $E^{\circ}\left(\mathrm{N}^{+} / \mathrm{N}\right)-E^{\circ}\left(\mathrm{M}^{+} /\right.$ $\mathrm{M})=E^{\circ}{ }_{N}-E^{\circ}{ }_{M}$, ou seja, quanto mais forte for o oxidante $N^{+}$(maior $E_{N}^{\circ}$ ) e mais forte for $\mathrm{O}$ redutor $\mathrm{M}$ (menor $E^{\mathrm{o}}{ }_{\mathrm{M}}$ ).

Para um mesmo oxidante $\mathrm{N}^{+}$, a reacção estará tanto mais deslocada quanto mais estabilizado por solvatação estiver o catião $\mathrm{M}^{+}$e é este o factor que acaba por ser dominante no comportamento do catião lítio em meio aquoso.
Embora todos os catiões $\mathrm{Li}^{+}, \mathrm{Na}^{+}, \mathrm{K}^{+}$, etc. tenham a mesma carga, o $\mathrm{Li}^{+}$é o mais estabilizado por solvatação $\left(\Delta H_{\text {hidrat }}^{\circ}=-520 \mathrm{~kJ} / \mathrm{mol}\right.$ [3] $)$ por ser o mais pequeno (a maior densidade de carga positiva torna mais fortes as ligações com as moléculas de água). Para o sódio e potássio estas entalpias são menores $(-405 \mathrm{e}-321 \mathrm{~kJ} /$ mol, respectivamente [3]).

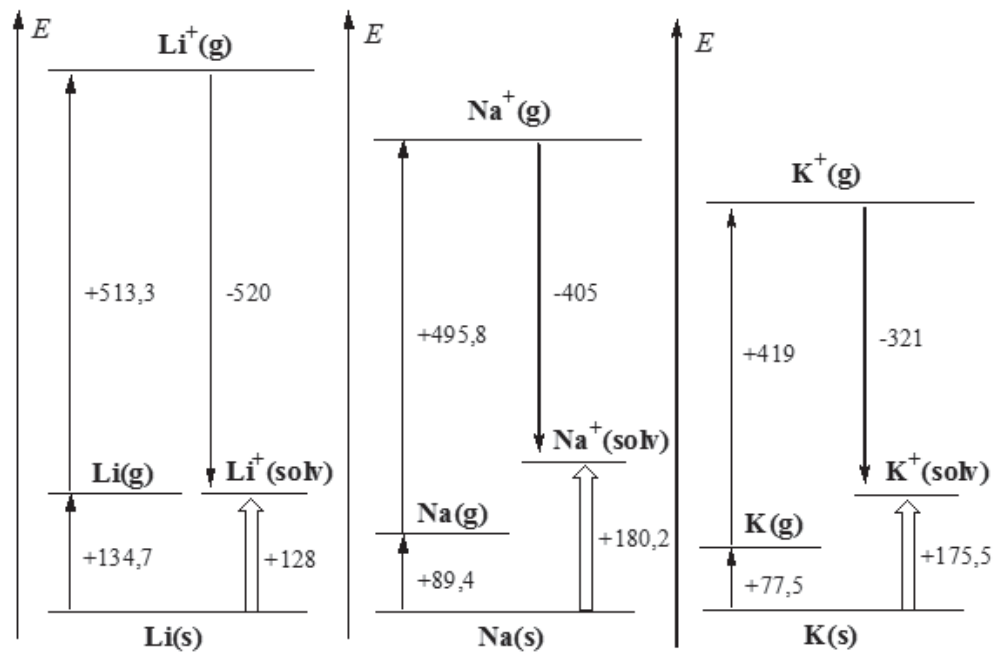

Assim, a reacção

$$
\mathrm{Li}(\mathrm{s})+\mathrm{N}^{+}(\mathrm{aq}) \rightarrow \mathrm{Li}^{+}(\mathrm{aq})+\mathrm{N}(\mathrm{s})
$$

está mais deslocada no sentido dos produtos do que as correspondentes reacções dos outros catiões alcalinos

$$
\begin{aligned}
\mathrm{Na}(\mathrm{s})+\mathrm{N}^{+}(\mathrm{aq}) & \rightarrow \mathrm{Na}^{+}(\mathrm{aq})+\mathrm{N}(\mathrm{s}) \\
\mathrm{K}(\mathrm{s})+\mathrm{N}^{+}(\mathrm{aq}) & \rightarrow \mathrm{K}^{+}(\mathrm{aq})+\mathrm{N}(\mathrm{s})
\end{aligned}
$$

de acordo com os correspondentes potenciais de eléctrodo.

\section{Catiões derivados do cobre [2b]}

A maior estabilidade do catião $\mathrm{Cu}(\mathrm{II})$ deve-se também a efeitos de solvatação. Além do catião $\mathrm{Cu}(\mathrm{II})$ ser mais pequeno que o catião $\mathrm{Cu}(\mathrm{I})$, tem ainda maior carga eléctrica (dupla). Não admira, assim, que o catião $\mathrm{Cu}$ (II) em água esteja mais fortemente hidratado $\left(\Delta H_{\text {hidrat }}^{\circ}=-2100 \mathrm{~kJ} / \mathrm{mol}\right.$ [3] $)$ do 


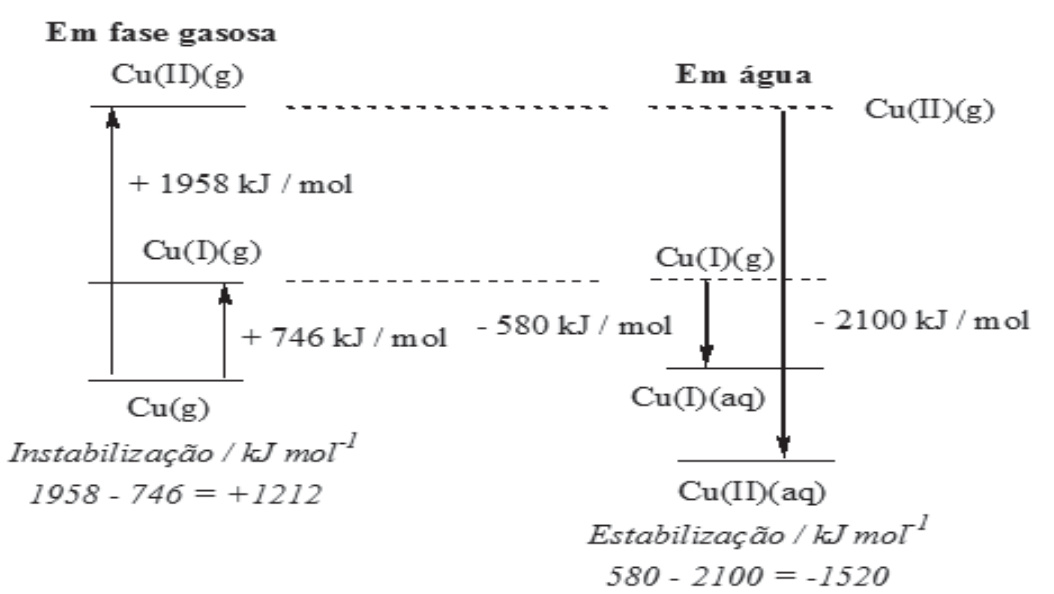

Em fase gasosa o catião $\mathrm{Cu}(\mathrm{I})$ é efectivamente mais estável. Pode existir em solução, sem que se dê a dismutação, desde que complexado por certos ligandos ( $\mathrm{CN}^{-}$, Piridilo).

\section{Catiões e aniões de metals alcalinos $[4,5]$}

Já nos anos 50 do século 20 eram conhecidos aniões de metais alcalinos em fase gasosa, mas só por volta de 1965 foi proposta a sua existência nas soluções azuis obtidas da dissolução de metais alcalinos em meio amoniacal (amoníaco líquido), juntamente com electrões solvatados:

$\mathrm{Na}(\mathrm{am}) \rightarrow \mathrm{Na}^{+}(\mathrm{am})+\mathrm{e}_{\text {solv }}^{-}(\mathrm{am})$ (em $\mathrm{NH}_{3}$ líquido)

$\mathrm{Na}(\mathrm{am})+\mathrm{e}_{\text {solv }}^{-}(\mathrm{am}) \rightarrow \mathrm{Na}^{-}(\mathrm{am})$ (em $\mathrm{NH}_{3}$ líquido)

$\mathrm{Na}$ presença de moléculas capazes de complexar fortemente os catiões dos metais alcalinos, como os chama-

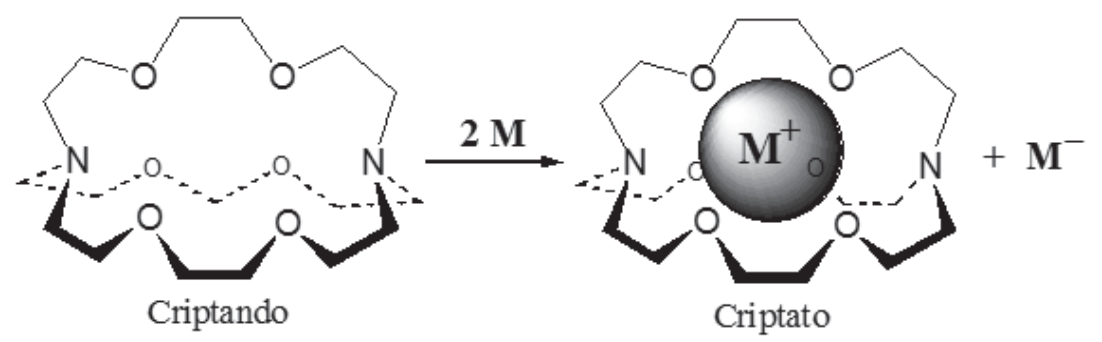
podem ser facilmente dissolvidos em vários solventes orgânicos.

A reacção de formação de catiões e aniões a partir de metais é deslocada no sentido da formação de aniões metálicos devido à complexação dos catiões:

$$
\begin{aligned}
& 2 \mathrm{M} \rightleftharpoons \mathrm{M}^{+}+\mathrm{M}^{-} \\
& \mathrm{M}^{+}+\text {Crip } \rightarrow \mathrm{M}^{+} \text {Crip }
\end{aligned}
$$

Em 1974, a partir de sódio metálico e do criptando da figura, foi isolado o primeiro composto sólido em que existem aniões $\mathrm{Na}^{-}$e catiões $\mathrm{Na}^{+}$, um composto cristalino com cristais em placas finas, hexagonais, e ponto de fusão $83^{\circ} \mathrm{C}$ : o $\mathrm{Na}^{+} \mathrm{CripNa}{ }^{-}$.

Posteriormente já foram preparados muitos outros compostos deste tipo a partir de metais alcalinos, alcalino-terrosos e outros.

Note-se que para se formar um anião derivado de um metal alcalino é necessário que uma partícula com baixa energia de ionização Ihe forneça o electrão, ou seja, que o electrão provenha de outro átomo metálico.

$\mathrm{Na}(\mathrm{am})+\mathrm{Na}(\mathrm{am}) \rightarrow \mathrm{Na}^{+}(\mathrm{am})+\mathrm{Na}^{-}(\mathrm{am})$

Em solução aquosa quem recebe o electrão é a molécula de água (muito mais oxidante), libertando-se hidrogénio:

$\mathrm{Na}(\mathrm{s})+\mathrm{H}_{2} \mathrm{O}(\mathrm{l}) \rightarrow \mathrm{Na}^{+}(\mathrm{aq})+1 / 2 \mathrm{H}_{2}(\mathrm{~g})+\mathrm{HO}^{-}(\mathrm{aq})$

A formação de anião hidreto em meio aquoso é também possível. Por exemplo, quando o hidrogénio atómico, em solução aquosa, reage com o electrão solvatado [6], liberta-se hidrogénio molecular

$\mathrm{H} \bullet+\mathrm{e}_{\text {solv }}^{-} \rightarrow \ldots \rightarrow \mathrm{H}_{2}(\mathrm{~g})$

através da formação de anião hidreto, que rapidamente reage com a água removendo um protão:

$$
\begin{aligned}
& \mathrm{H} \cdot+\mathrm{e}_{\text {solv }}^{-} \rightarrow \mathrm{H}^{-} \\
& \mathrm{H}^{-}+\mathrm{H}_{2} \mathrm{O}(\mathrm{l}) \rightarrow \mathrm{H}_{2}(\mathrm{~g})+\mathrm{HO}^{-}(\mathrm{aq})
\end{aligned}
$$

\section{CuRIOSIDADES HistóRICAS}

Grandes erros: «Todas as tentativas de aplicar métodos matemáticos no estudo de questões químicas é profundamente irracional e contrário ao espírito da química ... se a análise matemática alguma vez tiver um lugar proeminente em química - uma aberração que é felizmente quase impossível - daria lugar a uma rápida e generalizada degeneração desta ciência».

\section{REFERÊNCIAS}

[1] Tabela Periódica, SPQ.

[2] N. N. Greenwood e A. Earnshaw, "Chemistry of the Elements", Pergamon Press, 1984. a) 4.2.4 e 4.2.5; b) 28.2.4 e 28.3.

[3] P. W. Atkins, "Physical Chemistry", Oxford University Press, 4th ed. (1990) p.947.

[4] A. S. Ellaboudy, D. H. Holton, N. C. Pyper, P. P. Edwards, B. Wood e W. McFarlane, Nature 321 (1986) 684-685.

[5] James L. Dye, Angew. Chem.Int. Ed. Engl. 18 (1979) 587-598.

[6] C. A. Kelly e D. R. Rosseeinsky, Phys. Chem. Chem. Phys. 3 (2001) 2086-2090 .

Auguste Comte, Cours de Philosophie Positive, 1830. 